\title{
Blow Up Of \\ Quintic Power 1-D Nonlinear Schroedinger Equation
}

\author{
Agah D. Garnadi
}

\begin{abstract}
This work studies an adaptive finite difference approximation to the one dimensional nonlinear Schroedinger equiation with quintic power, with special emphasis on the case when the solution blows up with finite blowing-uptime $T_{\infty}$. The adaptivity is utilizing similarity scaling adaptive grids studied by Berger and Kohn to study numerical solution of semilinear heat equations with finite blowing-up time. Furthermore, we reports an asymptotic behavior of the blow-up solution approaching $T_{\infty}$ time singularity.
\end{abstract}

Keyworsds Nonlinear Schroedinger, critical power, finite time solution, blow up. 


\section{Implementation of Static Adaptive Grid Methods for Quintic Non-Linear Schrödinger Equation}

In this note we examines rescaling algorithms of Berger and Kohn [BK88]. There are two objectives in examining of this algorithms, first is to reverse engineered the algorithm to solve blow-up of solution for quintic non-linear Schrödinger, including its basic data structures that underlies the algorithms. The second objective is examining the behavior of solution when approaching singularity.

The basic reason to examine this algorithm is due to the fact that the NLS solution share the same scaling symmetry that also admitted by semi-linear parabolic equation. Furthermore, this symmetry is the crux in the Berger \& Kohn algorithm.

We consider problem of one-dimensional non-linear Schrödinger equation with critical power:

$$
u_{t}=i u_{x x}+i|u|^{4} u
$$

with given initial values:

$$
u(x, 0)=f(x)
$$

and prescribed Dirichlet boundary values:

$$
u(-1, t)=u(1, t)=0.0
$$

\section{Algorithm Description}

Let $u(x, t)$ be a solution of quintic Non-Linear Schrödinger, define:

$$
u_{\epsilon}(x, \tau)=\epsilon^{\frac{1}{2}} u\left(\epsilon x, \epsilon^{2} \tau\right), \epsilon>0
$$

If $u$ is defined for $-1 \leq x \leq 1$ and $0 \leq t<T$, then the domain of $u_{\mathrm{e}}$ is:

$$
-\epsilon^{-1} \leq x \leq \epsilon^{-1}, 0 \leq \tau \leq \epsilon^{2} T
$$

To determine the algorithm, three parameter must be fixed:

$$
\epsilon=\text { scaling factor }
$$

$M=$ Maximum before rescaling

$\alpha=$ control parameter determines interval width to be rescaled (i.e

$$
\left.\alpha M \leq|u|_{\infty} \leq M\right)
$$


These parameters should be chosen so that : $\epsilon^{-1}>1$ is a small integer, and $0<\alpha<1$.

Remark: Typical choice are $\epsilon^{-1}=4, M=\sqrt{8}, \alpha=\frac{1}{2}$ in [BK88].

Successively the algorithms compute a sequence of functions: $u_{k}\left(x_{k}, \tau_{k}\right)$ where:

$$
\begin{gathered}
u_{k}=\mathrm{k} \text {-th rescaled solution, } \\
x_{k}=\mathrm{k} \text {-th rescaled spatial variable, } \\
\tau_{k}=\text { clock measuring (rescaled) time for } u_{k},
\end{gathered}
$$

The initial index $k=0$ corrresponds to the 'real' solution $u$ and a function of 'real' space $x$ and 'real' time $t$ :

$$
u_{0}=u ; x_{0}=x ; \tau_{0}=t
$$

This initial index $k=0$, from now on we call base level. The first stage of the algorithms simply integrate the NLS equation at base level until the maximum amplitude surpassing the preset threshold $\mathrm{M}$ (This is under assumption that the initial data $\phi<M$, and satisfies $E(\phi)<0$.)

We choose Forward Euler for time differencing, while using center differencing for spatial variables:

$\frac{u\left(x_{j}, t_{n+1}\right)-u\left(x_{j}, t_{n}\right)}{\Delta t}=i \frac{\left(u\left(x_{j-1}, t_{n}\right)-2 u\left(x_{j}, t_{n}\right)+u\left(x_{j+1}, t_{n}\right)\right)}{\Delta t}+i\left|u\left(x_{j}, t_{n}\right)\right|^{4} u\left(x_{j}, t_{n}\right)$

Typical choice of time step is : $\Delta t=\left(\frac{h}{2}\right)^{2}$, where $h$ is initial spatial discretization at base level. The solution is integrated until the first-time step when $\left|u\left(-, t_{n}\right)\right|_{\infty}>M$ reached. By using two-time levels, calculate $\tau_{0}^{*}$ using linear interpolation with $t_{n}-\Delta t<\tau_{0}<t_{n}$ such that:

$$
\left|u\left(-, \tau_{0}^{*}\right)\right|_{\infty}=M
$$

The interval to be scaled at the next stage, $\left(x_{0}^{-}, x_{0}^{+}\right)$, essentially is the set of point where $\left|u\left(-, \tau_{0}^{*}\right)\right|_{\infty} \geq \alpha M$. For computational convenience, we choose for the end points of the interval $\left(x_{0}^{-}, x_{0}^{+}\right)$to be coarse points. By doing so, the end points are defined by:

$$
\left|u\left(x_{0}^{-}-h, \tau_{0}^{*}\right)\right|<\alpha M \leq\left|u\left(x_{0}^{-}, \tau_{0}^{*}\right)\right|
$$




$$
\left|u\left(x_{0}^{+}+h, \tau_{0}^{*}\right)\right|<\alpha M \leq\left|u\left(x_{0}^{+}, \tau_{0}^{*}\right)\right|
$$

The first 'rescaled solution' $u_{1}$ is related to $u$ by:

$$
u_{1}\left(x_{1}, \tau_{1}\right)=\epsilon^{\frac{1}{2}} u\left(\epsilon x_{1}, \tau_{0}^{*}+\epsilon^{2} \tau_{1}\right)
$$

We want $u$ to be evaluated on the right hand side only where $|u| \geq \alpha M$, therefore $x_{1}$ restricted to the interval:

$$
\epsilon^{-1} x_{0}^{-} \leq x_{1} \leq \epsilon^{-1} x_{0}^{+}
$$

The maximum value of amplitude $u_{1}$ at its initial time $\tau_{1}=0$, which corresponds to $t=\tau_{0}$, is reduced from: $\left|u\left(-, \tau_{0}^{*}\right)\right|_{\infty}=M$ to $|u(-, 0)|_{\infty}=$ $\epsilon^{\frac{1}{2}} M<M$, this is the purpose of rescaling. Due to the scale invariance property, $u_{1}$ solves the same equations as $u$, with respect to its (rescaled) 'space' and 'time' variables $x_{1}=\epsilon^{-1} x, \tau_{1}=\left(t-\tau_{0}^{*}\right) \epsilon^{2}$ :

$$
u_{\tau_{1}}=i u_{x_{1} x_{1}}+i|u|^{4} u
$$

So the numerical solver on the first stage still can be used without change to solve the first rescaled equation.

The calculation of $u_{1}$ requires initial data $u_{1}(-, 0)$ and bonndary data $u_{1}\left(\epsilon^{-1} x_{1}^{+-}, \tau\right)$. The initial data are easily available by rescaling $u$ :

$$
u_{1}\left(x_{1}, 0\right)=\epsilon^{\frac{1}{2}} u\left(\epsilon x_{1}, \tau_{0}^{*}\right)
$$

While the boundary data are obtained by coarse mesh approximation [ci71] method: i.e by using the previously computed over coarse mesh to determine boundary condition for the refined problem. Particularly, the right hand side:

$$
\begin{aligned}
& u_{1}\left(\epsilon x_{0}^{+}, N \Delta \tau_{1}\right)=\epsilon^{\frac{1}{2}} u\left(x_{0}^{+}, \tau_{0}^{*}+\epsilon^{2} N \Delta \tau_{1}\right) \\
& u_{1}\left(\epsilon x_{0}^{-}, N \Delta \tau_{1}\right)=\epsilon^{\frac{1}{2}} u\left(x_{0}^{-}, \tau_{0}^{*}+\epsilon^{2} N \Delta \tau_{1}\right)
\end{aligned}
$$

can be calculated using $\theta$-method to $u$ with a time step $\epsilon^{2} \Delta \tau_{1}$ ( which is smaller than $\Delta \tau_{0}=\Delta t$ ) used in calculation of $u$ on coarse grid, but only at boundary points $x_{0}^{-}$and $x_{0}^{+}$

By repeating the above procedures, we obtain the next stage calculation. So the $(k+1)$-th rescaled solution $u_{k+1}$ is introduced when $\tau_{k}$, the 'clock' for $u_{k}$, reaches a value $\tau_{k}^{*}$ such that:

$$
\left|u_{k}\left(-, \tau_{k}^{*}\right)\right|_{\infty}=M
$$


The interval in $x_{k}$-space to be recaled, $\left(x_{k}^{-}, x_{k}^{+}\right)$, consist precisely the region where $\left|u\left(-, \tau_{k}\right)\right|_{\infty} \geq \alpha M$ :

$$
\begin{aligned}
& \left|u\left(x_{k}^{-}-h_{k}, \tau_{k}^{*}\right)\right|_{\infty}<\alpha M \leq\left|u\left(x_{k}^{-}, \tau_{k}^{*}\right)\right|_{\infty} \\
& \left|u\left(x_{k}^{+}+h_{k}, \tau_{k}^{*}\right)\right|_{\infty}<\alpha M \leq\left|u\left(x_{k}^{+}, \tau_{k}^{*}\right)\right|_{\infty}
\end{aligned}
$$

The next rescaled solution $u_{k+1}$ is related to $u_{\epsilon}$ by:

$$
u_{k+1}\left(x_{k+1}, \tau_{k+1}\right)=\epsilon^{\frac{1}{2}} u_{\epsilon}\left(\epsilon x_{k+1}, \tau_{k}^{*}+\epsilon^{2} \tau_{k+1}\right)
$$

its 'rescaled space' and 'rescaled time' variables $x_{k+1}$ and $\tau_{k+1}$ tange over:

$$
\epsilon^{-1} x_{k}^{-} \leq x_{k+1} \leq \epsilon^{-1} x_{k}^{+}, \tau_{k+1} \geq 0
$$

Its initial data $u_{k+1}\left(x_{k+1}, 0\right)$ are determined by rescaling $u_{k}\left(-, \tau_{k}^{*}\right)$ using interpolation to define it on a refined spatial grid of width $h_{k+1}=h$. Its boundary data $u_{k+1}\left(\epsilon^{-1} x_{k}^{+-}, N \Delta \tau_{k+1}\right)$ are calculated using coarse mesh approximation from $u_{k}$ and it is stepped forward in time with time step $\Delta \tau_{k+1}=\Delta t$. Previously rescaled solutions are stepped independently: for illustration with choice $\epsilon=\frac{1}{4}$, then $u_{k}$ is stepped once for every 16 time steps of $u_{k+1}, u_{k-1}$ advanced once for every 256 time steps of $u_{k+1}$, etc.

Whenever a fine grid solution is computed at a point in space time where a coarser mesh also defined, the value of the coarse mesh solution is updated to agree with the finer grid calculation. When a time step is reached such that $\left|u_{k+1}\left(-, N \Delta \tau_{k+1}\right)\right|_{\infty}>M$, then it is time for another rescaling. A smaller time time $\tau_{k+1}^{*}$ is to be find such that $\left|u_{k+1}\left(-, \tau_{k+1}^{*}\right)\right|_{*}=M$, and the procedure is repeated.

\section{Numerical Experiment}

In our numerical experiment, we use initial data $\phi(x)=7.44 \cos \left(\frac{\pi}{2} x\right)$, which satisfy $E(\phi)<0$ and choose the following parameters : $=\frac{1}{2}, M=$ $(2 \sqrt{2})|u(-, 0)|_{\infty}$, and $\alpha=0.5$ for the algorithms. The values of spatial grid width $\Delta x=h$ and time step $\Delta t=k$ are chosen to be kept : $\Delta t=\left(\frac{\Delta x}{2}\right)^{2}$, and each run test reaches 31 level iterations. We did mumerical testing for $h=0.02$ and $h=0.01$ corresponds to 99 and 199 interior points respectively on the initial grid for $u(x, 0)=\phi(x)$.

The relationship between $\mathrm{k}$-th level solution $u_{k}\left(x_{k}, t_{k}\right)$ in terms to the actual solution $u(x, t)$ is as follows. If $\tau_{k}$ denotes the time at which $\mathrm{k}$-th level 
solution $u_{k}\left(x_{k}, t_{k}\right)$ needs to be rescaled to the next higher level $(k+1)$-th level solution $u_{k+1}\left(x_{k+1}, t_{k+1}\right)$, then :

$$
\tau_{k}=\tau_{0}^{*}+\epsilon^{2} \tau_{1}^{*}+\cdots+\epsilon^{2 k} \tau_{k}^{*}
$$

where $\tau_{j}^{*}$ is the computed time at which rescaling is performed for $u_{j}$ to the next level $u_{j+1}$. Iteration of scaling relationship between level also gives formula for current level computed solution $u_{k}$ at the time for rescaling to be performed:

$$
u_{k}\left(x_{k}, \tau_{k}^{*}\right)=\epsilon^{\frac{k}{2}} u\left(\epsilon^{k} x_{k}, \tau_{k}\right)
$$

where $x_{k}$ is the spatial variabel for $u_{k}$. Particularly, at $\tau_{k}$ the amplitudes of $u$ has multiplied by $\epsilon^{-\frac{k}{2}}$ :

$$
\left|u\left(-, \tau_{k}\right)\right|_{\infty}=\epsilon^{-\frac{k}{2}} M
$$

Hence the calculated blow-up time is $T_{\infty}=\lim _{k \rightarrow \infty} \tau_{k}$.

In Figures 1 the result for 99 interior points grid are portrayed. Fig. 1.a shows the scaled solution with the scaled abscisca stretched to $[-1,1]$ intervals which does not have any effects whatsoever to the solution. At a glance this result does not gives a clue about the oscillatory behaviour of the solution. This pictures is much more clearer if we take a look at fig 1.b which portrayed that the scaled solution that shown in fig 1.a is oscilating back and forth from lower graphs to upper graphs. Furthermore, the amplitude $\left|u_{k}\right|_{\infty}$ grows monotonically increasing. To see the effects of cutting levels for interpolation before regridding to the next higher level, we shows the result using cutting level $\alpha=0.40$ as shown in Figures 2 , it is clear that the solution is not significantly affected.

The results for 199 interior points grid are portrayed in figures 3 and 4 for $\alpha=0.5$ and 8.4 respectively. 
Fig. 1 : scaled solution using 99 interior points with $\alpha=0.50$

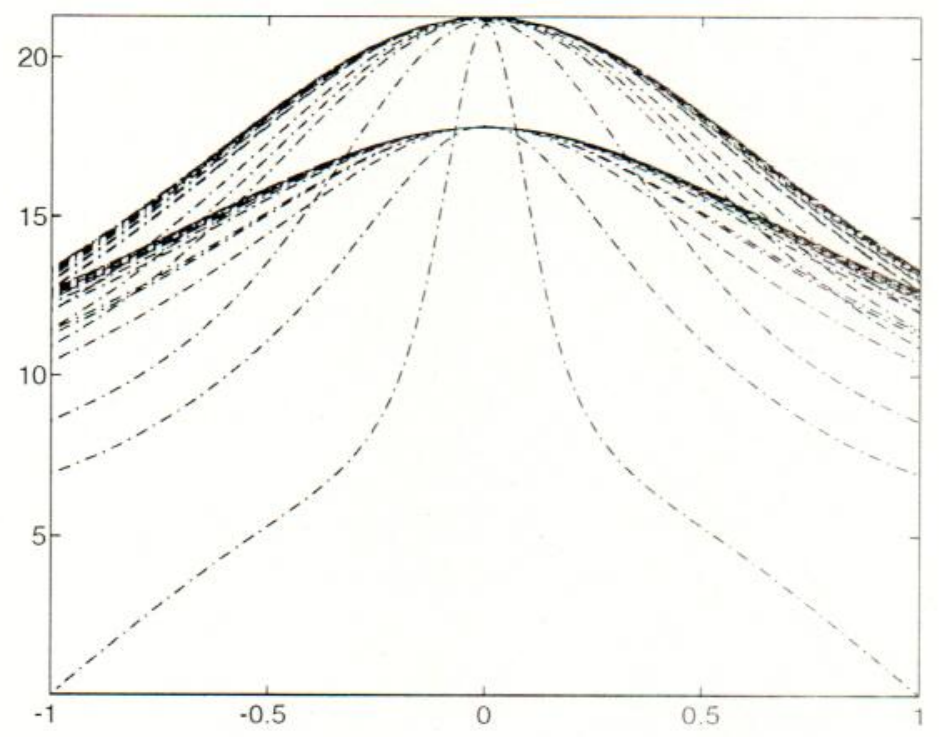

(a). Graphs of scaled solution at thresholding time

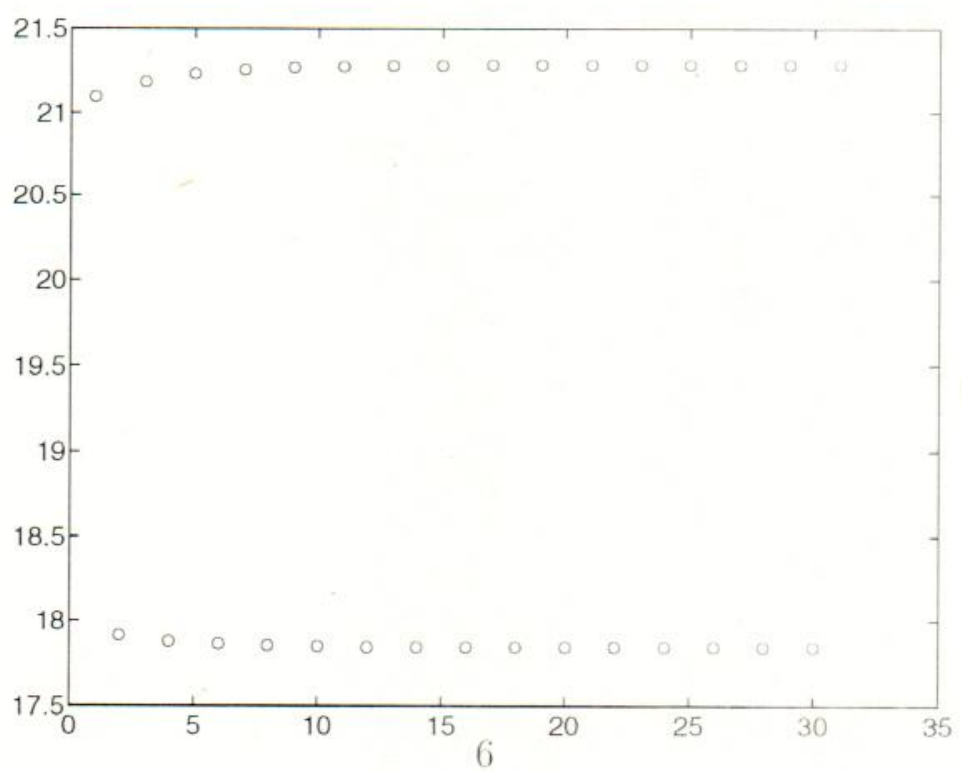

(b). amplitudes of scaled solution 
Fig. 2: scaled solution using 99 interior points with $\alpha=0.40$

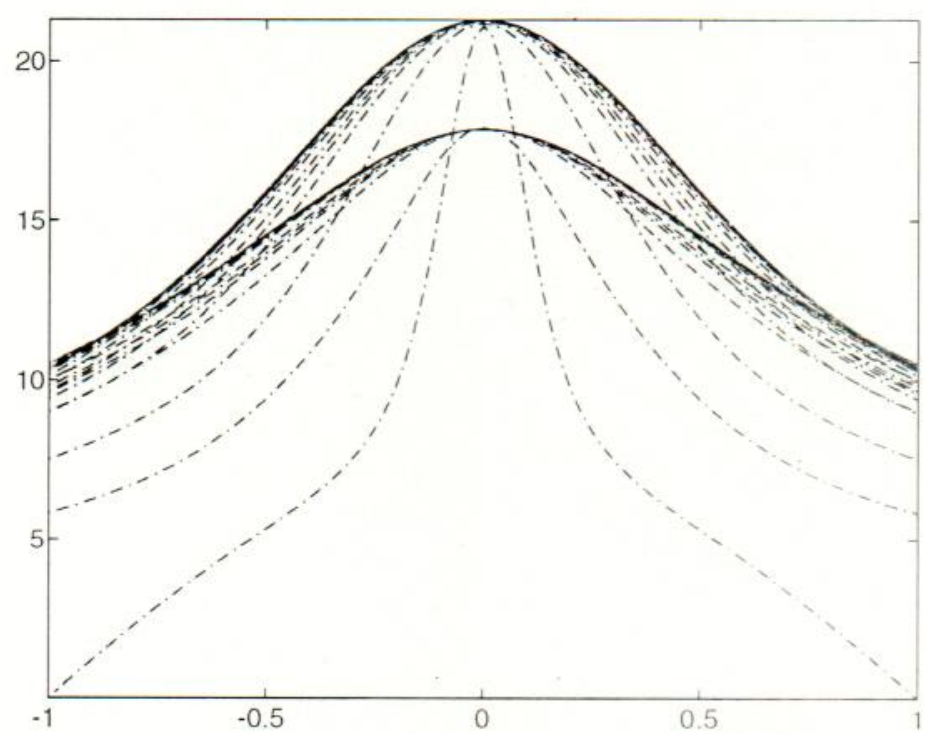

(a). Graphs of scaled solution at thresholding time

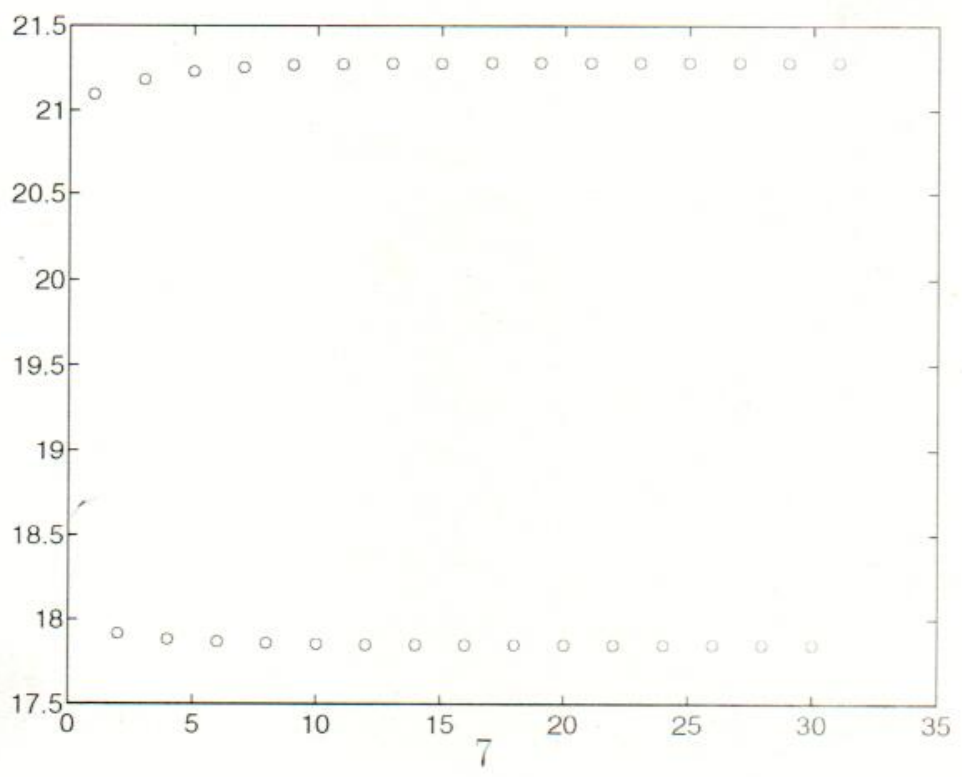

(b). amplitudes of scaled solution 
Fig. 3 : scaled solution using 199 interior points with $\alpha=0.50$

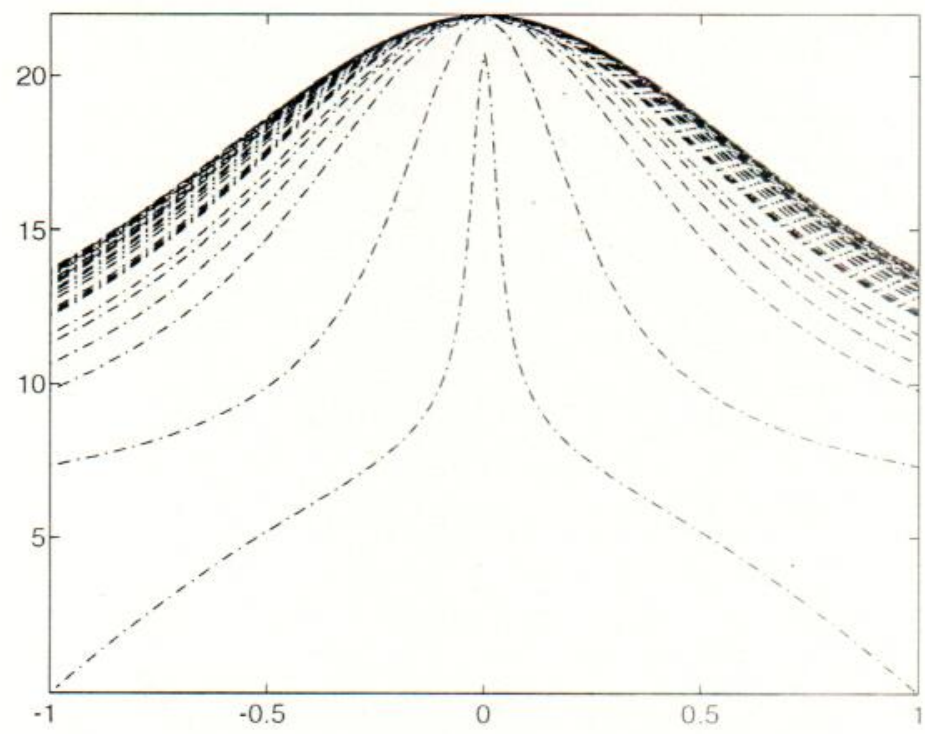

(a). Graphs of scaled solution at thresholding time

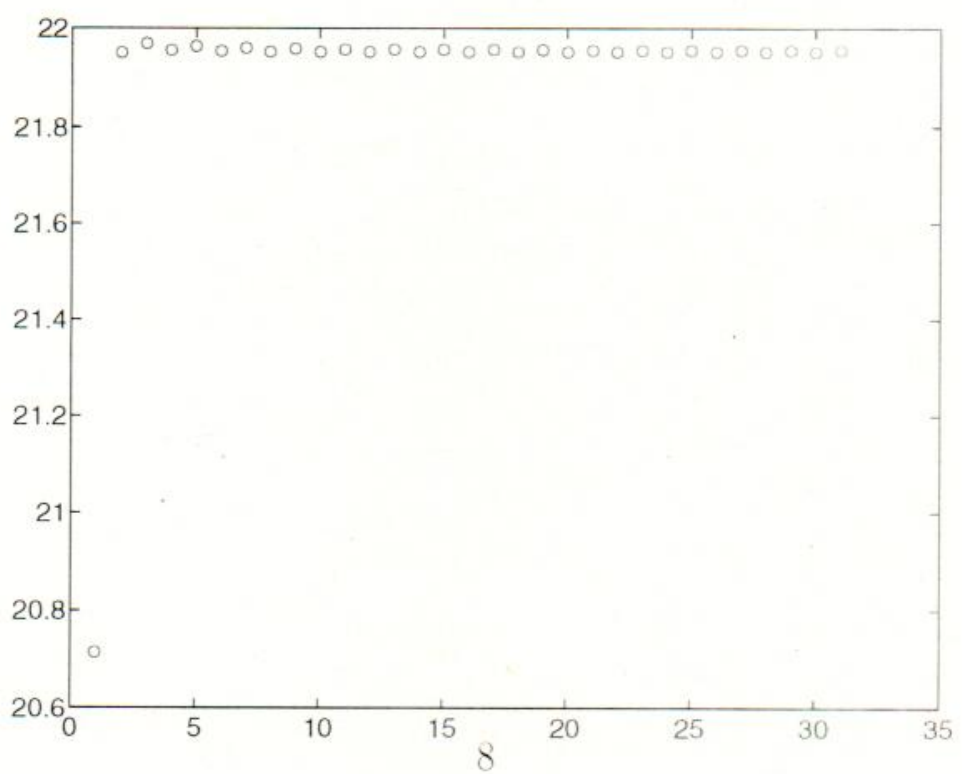

(b). amplitudes of scaled solution 
Fig. 4 : scaled solution using 199 interior points with $\alpha=0.40$

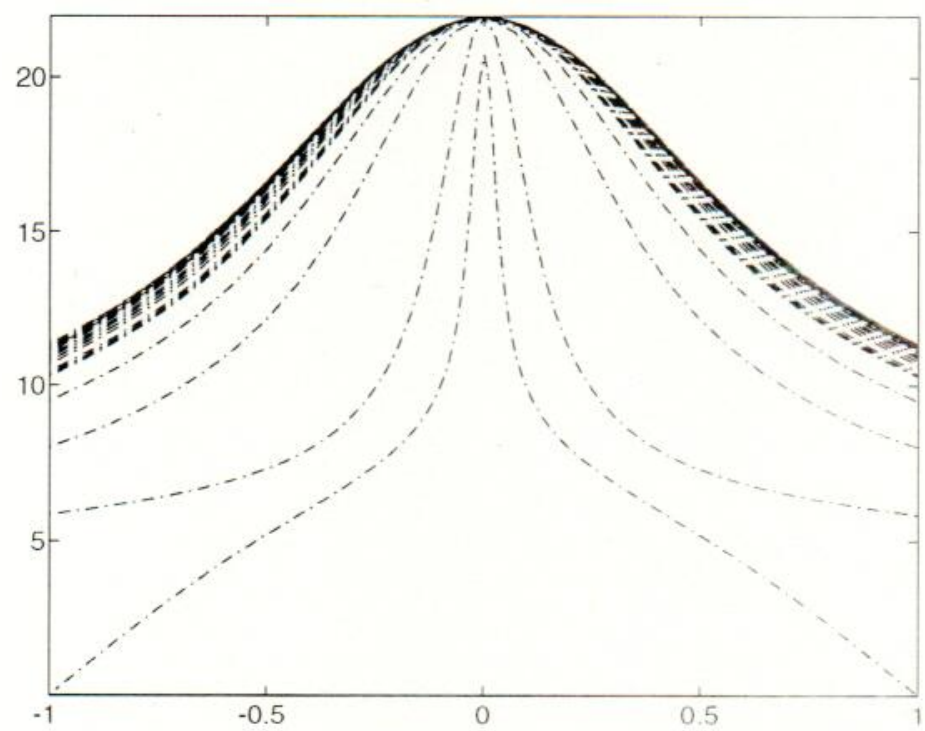

(a). Graphs of scaled solution at thresholding time

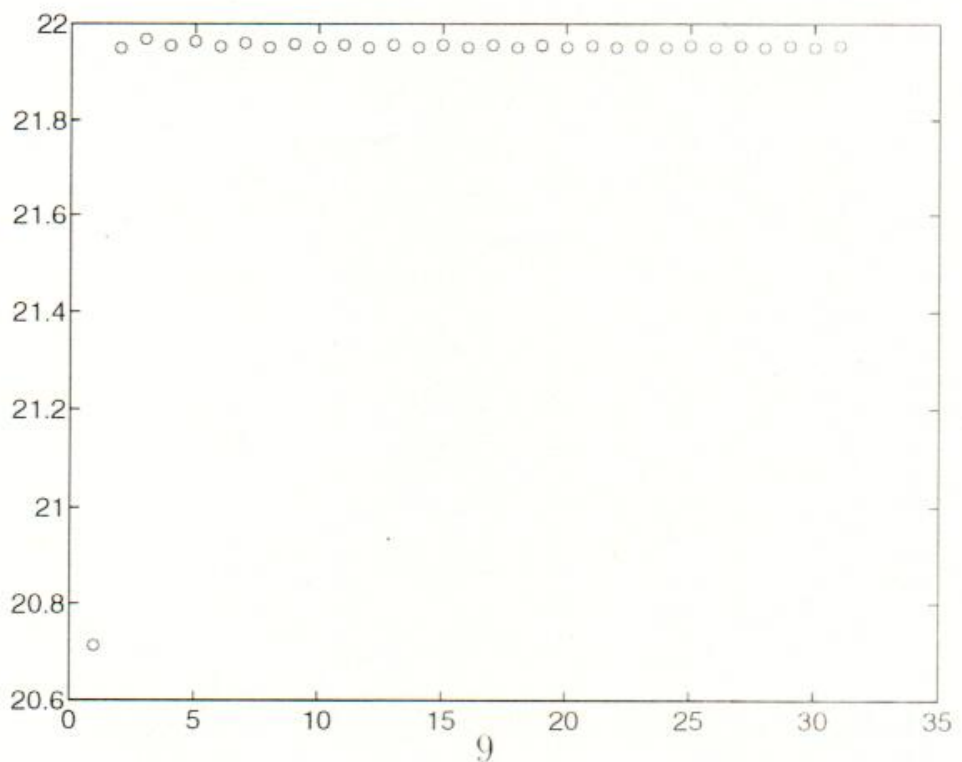

(b). amplitudes of scaled solution 


\section{Estimation of Blow-Up Parameters}

One of the concern is the behaviour of solution near blow up time $T_{\infty}$. On this occasion, we are more interested with the following conjectures about the growth of solution $u$ as approaching singularity:

$$
|u(x, t)|_{\infty} \infty\left(T_{\infty}-t\right)^{-\alpha}
$$

where parameters $T_{\infty}$ and $\alpha$ are parameter we are interested with.

The procedure we adopt in conjunction to rescaling is using non-linear least squares fit. In this procedures, if the discrete solutions sampled at times $t_{m_{j}}, j=1, \cdots, N$, then by minimising non-linear least squares:

$$
\sum_{j=1}^{N}\left[\log \left|u^{h}\right|_{\infty}-\log C^{h}-\alpha^{h} \log \left(T_{\infty}^{h}-t_{m,}\right)\right]^{2}
$$

with respect to $\log C^{h}$ (where $C^{h}$ is proportional constant in the growth of solution approaching singularity), $\alpha^{h}$ and $T_{\infty}^{h}$ to obtain the desired estimate of parameter.

We may use Levenberg-Marquardt algorithm or direct minimisation method to solve this problem. These algorithms is available in matlab routine form from public contribution at ftp.mathworks.com .

Both type of methods require initialisation to get it start. It is well known that the behavior to convergence of the methods is depends on initial starting points. To overcome this problem, the search for good starting point possibly we can do it naively by plotting $\log \left(\left|u_{k}\right|_{\infty}\right.$ vs $\log \left(T \infty-t_{k}\right)$. Since $T_{\infty}$ is not available, we may take as a guess by set it as: $T_{\infty}^{*}=m a x_{k}\left\{t_{k}\right\}$. This plots is shown in figure 5. From this plot, using guessing by eye, that we may choose as the starting points for the non-linear least squares are

$$
\alpha=-\frac{1}{4}, \log C^{h}=-3, T_{\infty}=\max _{k}\left\{t_{k}\right\}
$$

From the graphs, we get hints that the rate of numerical blow-up is identical. If we take a look at Table 1 and 2, the last 6 and 8 rows respectively shows an identical time, this indicated that in doing summation from 'scaled' time to the real time, the scaled time in the last coupled of steps is already too small. 


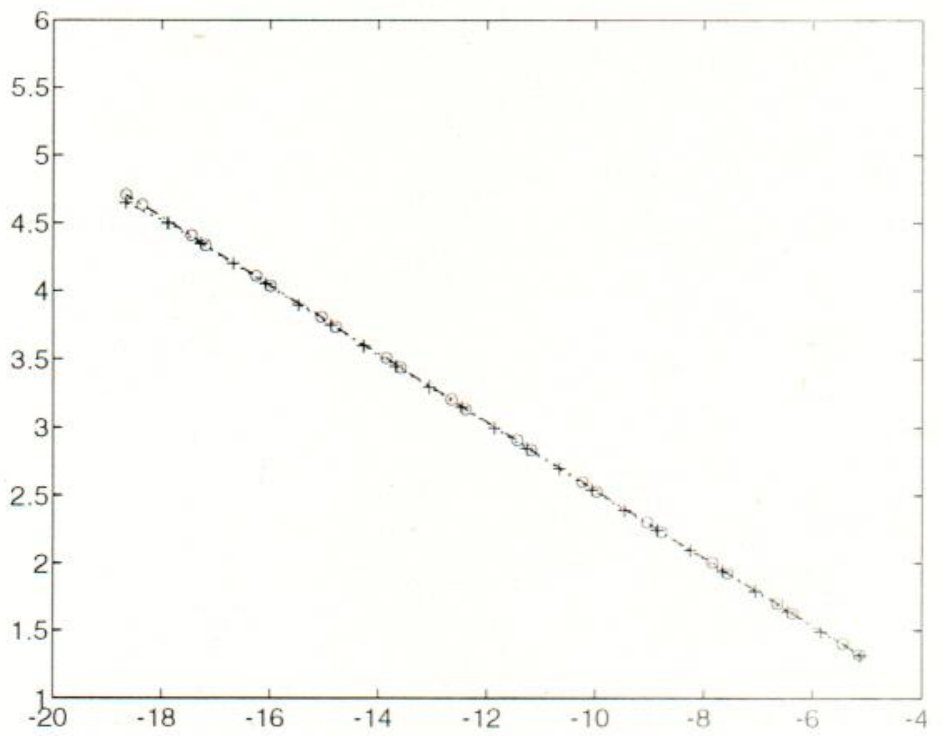

Fig. 5: Plots of $\log \left|u\left(-, t_{k}\right)\right|_{\infty}$ vs $\log \left(\max _{k}\left\{t_{k}\right\}-t_{k}\right)$ for $\alpha=0.5$ with legends $(+)$ for 199 interior points and $(o)$ for 99 interior points 


\section{Reference}

[BK88] Berger, M. and Kohn, R.V., 1988. A rescaling algorithm for the numerical calculation of blowing-up solutions. Communications on pure and applied mathematics, 41(6), pp.841-863. 
APPENDIX 


\begin{tabular}{|l|l|}
\hline Real Time & Amplitudes \\
\hline $4.998887132705376 \mathrm{e}-04$ & $1.324197431132756 \mathrm{e}+00$ \\
$5.032506577141724 \mathrm{e}-04$ & $1.403767082182199 \mathrm{e}+00$ \\
$5.065037400528967 \mathrm{e}-04$ & $1.627016729123788 \mathrm{e}+00$ \\
$5.067062272525857 \mathrm{e}-04$ & $1.703964306661082 \mathrm{e}+00$ \\
$5.069123984453981 \mathrm{e}-04$ & $1.929035011686420 \mathrm{e}+00$ \\
$5.069247981529175 \mathrm{e}-04$ & $2.004549553525023 \mathrm{e}+00$ \\
$5.069377793809985 \mathrm{e}-04$ & $2.230586457725435 \mathrm{e}+00$ \\
$5.069385460558265 \mathrm{e}-04$ & $2.305351605681715 \mathrm{e}+00$ \\
$5.069393604546764 \mathrm{e}-04$ & $2.531882669062714 \mathrm{e}+00$ \\
$5.069394081088580 \mathrm{e}-04$ & $2.606267145080100 \mathrm{e}+00$ \\
$5.069394591054332 \mathrm{e}-04$ & $2.833046134044841 \mathrm{e}+00$ \\
$5.069394620756115 \mathrm{e}-04$ & $2.907240251638687 \mathrm{e}+00$ \\
$5.069394652659041 \mathrm{e}-04$ & $3.134142434742920 \mathrm{e}+00$ \\
$5.069394654512859 \mathrm{e}-04$ & $3.208242109226244 \mathrm{e}+00$ \\
$5.069394656507723 \mathrm{e}-04$ & $3.435205224996833 \mathrm{e}+00$ \\
$5.069394656623508 \mathrm{e}-04$ & $3.509258218150447 \mathrm{e}+00$ \\
$5.069394656748216 \mathrm{e}-04$ & $3.736251409144335 \mathrm{e}+00$ \\
$5.069394656755450 \mathrm{e}-04$ & $3.810281366345998 \mathrm{e}+00$ \\
$5.069394656763245 \mathrm{e}-04$ & $4.037289390053447 \mathrm{e}+00$ \\
$5.069394656763697 \mathrm{e}-04$ & $4.111307986232018 \mathrm{e}+00$ \\
$5.069394656764184 \mathrm{e}-04$ & $4.338323324046790 \mathrm{e}+00$ \\
$5.069394656764212 \mathrm{e}-04$ & $4.412336317425833 \mathrm{e}+00$ \\
$5.069394656764243 \mathrm{e}-04$ & $4.639355262422727 \mathrm{e}+00$ \\
$5.069394656764245 \mathrm{e}-04$ & $4.713365492155751 \mathrm{e}+00$ \\
$5.069394656764247 \mathrm{e}-04$ & $4.940386216697989 \mathrm{e}+00$ \\
$5.069394656764247 \mathrm{e}-04$ & $5.014395082771604 \mathrm{e}+00$ \\
$5.069394656764247 \mathrm{e}-04$ & $5.241416685564428 \mathrm{e}+00$ \\
$5.069394656764247 \mathrm{e}-04$ & $5.315424878500377 \mathrm{e}+00$ \\
$5.069394656764247 \mathrm{e}-04$ & $5.542446914917382 \mathrm{e}+00$ \\
$5.069394656764247 \mathrm{e}-04$ & $5.616454775430620 \mathrm{e}+00$ \\
$5.069394656764247 \mathrm{e}-04$ & $5.843477026039515 \mathrm{e}+00$ \\
\hline
\end{tabular}




\begin{tabular}{|l|l|}
\hline Real Time & Amplitudes \\
\hline $1.075154094209945 \mathrm{e}-03$ & $1.316277113101874 \mathrm{e}+00$ \\
$1.081273995834679 \mathrm{e}-03$ & $1.491946516240474 \mathrm{e}+00$ \\
$1.082356263300261 \mathrm{e}-03$ & $1.642849546085317 \mathrm{e}+00$ \\
$1.082625074982046 \mathrm{e}-03$ & $1.793083271437194 \mathrm{e}+00$ \\
$1.082692519429425 \mathrm{e}-03$ & $1.943751106920740 \mathrm{e}+00$ \\
$1.082709321971602 \mathrm{e}-03$ & $2.094075626656857 \mathrm{e}+00$ \\
$1.082713534504286 \mathrm{e}-03$ & $2.244728947674374 \mathrm{e}+00$ \\
$1.082714584498448 \mathrm{e}-03$ & $2.395080103148426 \mathrm{e}+00$ \\
$1.082714847688655 \mathrm{e}-03$ & $2.545728093888006 \mathrm{e}+00$ \\
$1.082714913309244 \mathrm{e}-03$ & $2.696097452115047 \mathrm{e}+00$ \\
$1.082714929754683 \mathrm{e}-03$ & $2.846738363899495 \mathrm{e}+00$ \\
$1.082714933855990 \mathrm{e}-03$ & $2.997122397620984 \mathrm{e}+00$ \\
$1.082714934883650 \mathrm{e}-03$ & $3.147755118818826 \mathrm{e}+00$ \\
$1.082714935139992 \mathrm{e}-03$ & $3.298151521582571 \mathrm{e}+00$ \\
$1.082714935204212 \mathrm{e}-03$ & $3.448775733584751 \mathrm{e}+00$ \\
$1.082714935220234 \mathrm{e}-03$ & $3.599182850884776 \mathrm{e}+00$ \\
$1.082714935224248 \mathrm{e}-03$ & $3.749798693426087 \mathrm{e}+00$ \\
$1.082714935225249 \mathrm{e}-03$ & $3.900215273946848 \mathrm{e}+00$ \\
$1.082714935225500 \mathrm{e}-03$ & $4.050823123463213 \mathrm{e}+00$ \\
$1.082714935225563 \mathrm{e}-03$ & $4.201248173812378 \mathrm{e}+00$ \\
$1.082714935225578 \mathrm{e}-03$ & $4.351848516049984 \mathrm{e}+00$ \\
$1.082714935225582 \mathrm{e}-03$ & $4.502281213876786 \mathrm{e}+00$ \\
$1.082714935225583 \mathrm{e}-03$ & $4.652874573311372 \mathrm{e}+00$ \\
$1.082714935225583 \mathrm{e}-03$ & $4.803314215191963 \mathrm{e}+00$ \\
$1.082714935225583 \mathrm{e}-03$ & $4.953901117055937 \mathrm{e}+00$ \\
$1.082714935225583 \mathrm{e}-03$ & $5.104347086855792 \mathrm{e}+00$ \\
$1.082714935225583 \mathrm{e}-03$ & $5.254928037533380 \mathrm{e}+00$ \\
$1.082714935225583 \mathrm{e}-03$ & $5.405379786734268 \mathrm{e}+00$ \\
$1.082714935225583 \mathrm{e}-03$ & $5.555955264369508 \mathrm{e}+00$ \\
$1.082714935225583 \mathrm{e}-03$ & $5.706412299383357 \mathrm{e}+00$ \\
$1.082714935225583 \mathrm{e}-03$ & $5.856982750091334 \mathrm{e}+00$ \\
\hline
\end{tabular}




\begin{tabular}{|l|l|l|}
\hline $\log C^{h}$ & $\alpha^{h}$ & $T_{\infty}^{h}$ \\
\hline $2.548313421936826 \mathrm{e}-01$ & $-2.685704325526579 \mathrm{e}-01$ & $5.071656458422434 \mathrm{e}-04$ \\
$2.548313421936826 \mathrm{e}-01$ & $-2.685704325526579 \mathrm{e}-01$ & $5.071656458422434 \mathrm{e}-04$ \\
$2.548313421936826 \mathrm{e}-01$ & $-2.685704325526579 \mathrm{e}-01$ & $5.071656458422434 \mathrm{e}-04$ \\
$2.478305922873232 \mathrm{e}-01$ & $-2.936621768876075 \mathrm{e}-01$ & $5.071483470382586 \mathrm{e}-04$ \\
$2.478305922873232 \mathrm{e}-01$ & $-2.936621768876075 \mathrm{e}-01$ & $5.071483470382586 \mathrm{e}-04$ \\
$2.478305922873232 \mathrm{e}-01$ & $-2.936621768876075 \mathrm{e}-01$ & $5.071483470382586 \mathrm{e}-04$ \\
$-8.838383220043406 \mathrm{e}-01$ & $-4.953901685229035 \mathrm{e}-01$ & $5.071036006710695 \mathrm{e}-04$ \\
$-8.838383220043406 \mathrm{e}-01$ & $-4.953901685229035 \mathrm{e}-01$ & $5.071036006710695 \mathrm{e}-04$ \\
$-8.838383220043406 \mathrm{e}-01$ & $-4.953901685229035 \mathrm{e}-01$ & $5.071036006710695 \mathrm{e}-04$ \\
$-8.838383220043406 \mathrm{e}-01$ & $-4.953901685229035 \mathrm{e}-01$ & $5.071036006710695 \mathrm{e}-04$ \\
$-8.838383220043406 \mathrm{e}-01$ & $-4.953901685229035 \mathrm{e}-01$ & $5.071036006710695 \mathrm{e}-04$ \\
$-8.838383220043406 \mathrm{e}-01$ & $-4.953901685229035 \mathrm{e}-01$ & $5.071036006710695 \mathrm{e}-04$ \\
$-1.026749971202676 \mathrm{e}+00$ & $-6.055763480435493 \mathrm{e}-01$ & $5.072346716716340 \mathrm{e}-04$ \\
$-1.026749971202676 \mathrm{e}+00$ & $-6.055763480435493 \mathrm{e}-01$ & $5.072346716716340 \mathrm{e}-04$ \\
$-1.026749971202676 \mathrm{e}+00$ & $-6.055763480435493 \mathrm{e}-01$ & $5.072346716716340 \mathrm{e}-04$ \\
$-2.318515933797081 \mathrm{e}+00$ & $-8.154205421947027 \mathrm{e}-01$ & $5.071779235325483 \mathrm{e}-04$ \\
$-2.318515933797081 \mathrm{e}+00$ & $-8.154205421947027 \mathrm{e}-01$ & $5.071779235325483 \mathrm{e}-04$ \\
$-2.318515933797081 \mathrm{e}+00$ & $-8.154205421947027 \mathrm{e}-01$ & $5.071779235325483 \mathrm{e}-04$ \\
$-2.300979366207932 \mathrm{e}+00$ & $-8.214597554926192 \mathrm{e}-01$ & $5.072116880489871 \mathrm{e}-04$ \\
$-2.169546342780948 \mathrm{e}+00$ & $-8.394781196936152 \mathrm{e}-01$ & $5.070588196089297 \mathrm{e}-04$ \\
$-2.169546342780948 \mathrm{e}+00$ & $-8.394781196936152 \mathrm{e}-01$ & $5.070588196089297 \mathrm{e}-04$ \\
$-2.169546342780948 \mathrm{e}+00$ & $-8.394781196936152 \mathrm{e}-01$ & $5.070588196089297 \mathrm{e}-04$ \\
$-2.169546342780948 \mathrm{e}+00$ & $-8.394781196936152 \mathrm{e}-01$ & $5.070588196089297 \mathrm{e}-04$ \\
\hline
\end{tabular}




\begin{tabular}{|l|l|l|}
\hline $\log C^{h}$ & $\alpha^{h}$ & $T_{\infty}^{h}$ \\
\hline $1.363418403829975 \mathrm{e}-01$ & $-2.980806371370762 \mathrm{e}-01$ & $1.083144716101075 \mathrm{e}-03$ \\
$1.363418403829975 \mathrm{e}-01$ & $-2.980806371370762 \mathrm{e}-01$ & $1.083144716101075 \mathrm{e}-03$ \\
$1.363418403829975 \mathrm{e}-01$ & $-2.980806371370762 \mathrm{e}-01$ & $1.083144716101075 \mathrm{e}-03$ \\
$-8.908293715301646 \mathrm{e}-02$ & $-3.796872269625615 \mathrm{e}-01$ & $1.083348394285553 \mathrm{e}-03$ \\
$-8.908293715301646 \mathrm{e}-02$ & $-3.796872269625615 \mathrm{e}-01$ & $1.083348394285553 \mathrm{e}-03$ \\
$-1.047592011816777 \mathrm{e}-01$ & $-4.003137171869909 \mathrm{e}-01$ & $1.083196748581767 \mathrm{e}-03$ \\
$-1.047592011816777 \mathrm{e}-01$ & $-4.003137171869909 \mathrm{e}-01$ & $1.083196748581767 \mathrm{e}-03$ \\
$-1.047592011816777 \mathrm{e}-01$ & $-4.003137171869909 \mathrm{e}-01$ & $1.083196748581767 \mathrm{e}-03$ \\
$-1.047592011816777 \mathrm{e}-01$ & $-4.003137171869909 \mathrm{e}-01$ & $1.083196748581767 \mathrm{e}-03$ \\
$-1.057925182895881 \mathrm{e}+00$ & $-6.062489184601757 \mathrm{e}-01$ & $1.083263102014120 \mathrm{e}-03$ \\
$-1.057925182895881 \mathrm{e}+00$ & $-6.062489184601757 \mathrm{e}-01$ & $1.083263102014120 \mathrm{e}-03$ \\
$-1.057925182895881 \mathrm{e}+00$ & $-6.062489184601757 \mathrm{e}-01$ & $1.083263102014120 \mathrm{e}-03$ \\
$-1.057925182895881 \mathrm{e}+00$ & $-6.062489184601757 \mathrm{e}-01$ & $1.083263102014120 \mathrm{e}-03$ \\
$-1.020077504593948 \mathrm{e}+00$ & $-6.266859894780078 \mathrm{e}-01$ & $1.082890831624982 \mathrm{e}-03$ \\
$-1.020077504593948 \mathrm{e}+00$ & $-6.266859894780078 \mathrm{e}-01$ & $1.082890831624982 \mathrm{e}-03$ \\
$-1.020077504593948 \mathrm{e}+00$ & $-6.266859894780078 \mathrm{e}-01$ & $1.082890831624982 \mathrm{e}-03$ \\
$-1.020077504593948 \mathrm{e}+00$ & $-6.266859894780078 \mathrm{e}-01$ & $1.082890831624982 \mathrm{e}-03$ \\
$-1.020077504593948 \mathrm{e}+00$ & $-6.266859894780078 \mathrm{e}-01$ & $1.082890831624982 \mathrm{e}-03$ \\
$-1.020077504593948 \mathrm{e}+00$ & $-6.266859894780078 \mathrm{e}-01$ & $1.082890831624982 \mathrm{e}-03$ \\
$-1.020077504593948 \mathrm{e}+00$ & $-6.266859894780078 \mathrm{e}-01$ & $1.082890831624982 \mathrm{e}-03$ \\
$-1.020077504593948 \mathrm{e}+00$ & $-6.266859894780078 \mathrm{e}-01$ & $1.082890831624982 \mathrm{e}-03$ \\
$-1.020077504593948 \mathrm{e}+00$ & $-6.266859894780078 \mathrm{e}-01$ & $1.082890831624982 \mathrm{e}-03$ \\
$-1.020077504593948 \mathrm{e}+00$ & $-6.266859894780078 \mathrm{e}-01$ & $1.082890831624982 \mathrm{e}-03$ \\
\hline
\end{tabular}

